\title{
Rare isolated coracoid mid-process fracture with displacement in an adolescent: Case report
}

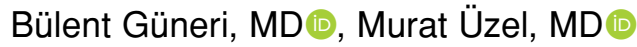 \\ Department of Orthopedics and Traumatology, Kahramanmaraş Sütçü İmam University, Faculty of Medicine, Kahramanmaraş, Turkey
}

Coracoid fractures are unusual injuries which are frequently accompanied by additional shoulder girdle injuries including dislocation of acromioclavicular and glenohumeral joints, fractures of clavicle, proximal humerus and scapular body. ${ }^{[1-3]}$ Isolated coracoid fractures (ICFs) are quite rare and can be

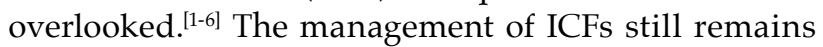
controversial among orthopedic surgeons..$^{[1,4-6]}$ In this article, we report an initially missed isolated coracoid mid-process fracture with displacement in an adolescent, treated successfully by Velpeau sling application. ${ }^{[7]}$

\section{CASE REPORT}

A 12-year-old male patient was admitted to the clinic due to mild pain on anterior aspect of his right shoulder with mild limitation in active motion. Medical history of the patient was uneventful except falling to right side while playing football three days before admission. He recalled hitting the lateral

Received: October 18, 2020

Accepted: February 05, 2020

Published online: September 11, 2020

Correspondence: Bülent Güneri, MD. Kahramanmaraş Sütçü İmam Üniversitesi Sağlık Uygulama ve Araștırma Hastanesi Ortopedi ve Travmatoloji Kliniği, 46040 Onikişubat, Kahramanmaraş, Turkey.

E-mail: bulentguneri@yahoo.com

Doi: $10.5606 /$ ehc. 2020.72842

Citation: Güneri B, Üzel M. Rare isolated coracoid mid-process fracture with displacement in an adolescent: Case report. Jt Dis Relat Surg 2020;31(3):630-633.

(2020 All right reserved by the Turkish Joint Diseases Foundation

This is an open access article under the terms of the Creative Commons Attribution-NonCommercial License, which permits use, distribution and reproduction in any medium, provided the original work is properly cited and is not used for commercial purposes (http://creativecommons.org/licenses/by-nc/4.0/).

\section{ABSTRACT}

Isolated coracoid fractures (ICFs) are rare and the management is controversial. In this article, we report a displaced ICF, treated conservatively with success. A 12-year-old male patient presented with mild pain in his right shoulder after simple fall. Physical examination was normal except mild tenderness on the coracoid process and mildly limited active shoulder motion. Plain radiographs did not demonstrate any apparent finding of pathology. Computed tomography (CT) images revealed isolated coracoid mid-process fracture with displacement. Significant symptomatic relief as well as sufficient callus formation, confirmed by follow-up CT examination, was achieved after Velpeau sling use for four weeks. After a follow-up duration of 14 months, excellent clinical and radiologic outcomes were accomplished. The result of this case supports the efficiency of conservative treatment for ICFs in adolescents, even in the presence of considerable fracture displacement. Computed tomography imaging is a valuable diagnostic tool for the assessment of ICF.

Keywords: Adolescent, bone, conservative treatment, coracoid process, fractures, scapula.

aspect of his right shoulder to the ground and crepitation which occurred for once while selfexamining shoulder motion in the direction of forward flexion. The patient had been admitted to the emergency department of a hospital on the same day of injury and informed to have soft tissue injury. Analgesic medication without any external support was given. A written informed consent was obtained from the legal guardian of the patient.

No unusual finding could be identified on inspection. Mild-to-moderate pain could be elicited with palpation on the coracoid process. Crepitus was not identified upon palpation as well as during active and passive shoulder motion. The range of forward flexion, extension, abduction, adduction, external rotation and internal rotation were $0-170,0-20,0-160$, $0-40,0-20$ and $0-90$ degrees on the right shoulder, respectively, while the range of forward flexion, 
extension, abduction, adduction, external and internal rotations were $0-180,0-50,0-170,0-60,0-40$ and $0-90$ degrees on the left shoulder, respectively. The patient mentioned mild aggravation of pain during active motion of the right shoulder. Active elbow flexionextension was within the range of 0-150 degrees for both sides. Neurological evaluation was normal. Bilateral shoulder radiographs did not demonstrate any apparent finding of pathology. With respect to history of previous trauma, computed tomography (CT) images were obtained and revealed isolated coracoid mid-process fracture (Eyres type 2) with considerable anteroinferior displacement of the distal fragment (Figure 1). ${ }^{[2,6]}$ Conservative management including Velpeau sling use and analgesic medication were opted for the patient.

Physical and radiologic examinations were performed on each outpatient follow-up visit. The patient reported significant relief with Velpeau sling use two weeks after admission. At the end of fourth week, he was almost pain-free during rest as well as active and passive shoulder motion. The follow-up $\mathrm{CT}$ examination, obtained at the end of fourth week, confirmed significant callus formation (Figure 2). With respect to callus formation and vanished symptoms, Velpeau sling was discontinued and active shoulder motions were allowed with restrictions from weightlifting for four weeks. Physical examinations and plain radiographs could not identify any remarkable finding during follow-up. Until he fell to his right side from a couch at $11^{\text {th }}$ month of follow-up, the patient was symptom-free in daily activities including weightlifting and sport activities. Upon this incident, he reported pain and limitation in the right shoulder motion. No additional finding could be detected on examination. Radiologic examination including $\mathrm{CT}$, which proved complete osseous union with disappearance of coracoid fracture line, did not reveal any pathologic finding (Figure 3). The patient was completely symptom-free on the last visit which was 14 months after the diagnosis of fracture and the Disabilities of the Arm, Shoulder and Hand score was 0 .

\section{DISCUSSION}

Coracoid fractures constitute $3-13 \%$ of scapular fractures and approximately $1 \%$ of all fractures in humans. ${ }^{[3]}$ These fractures frequently occur
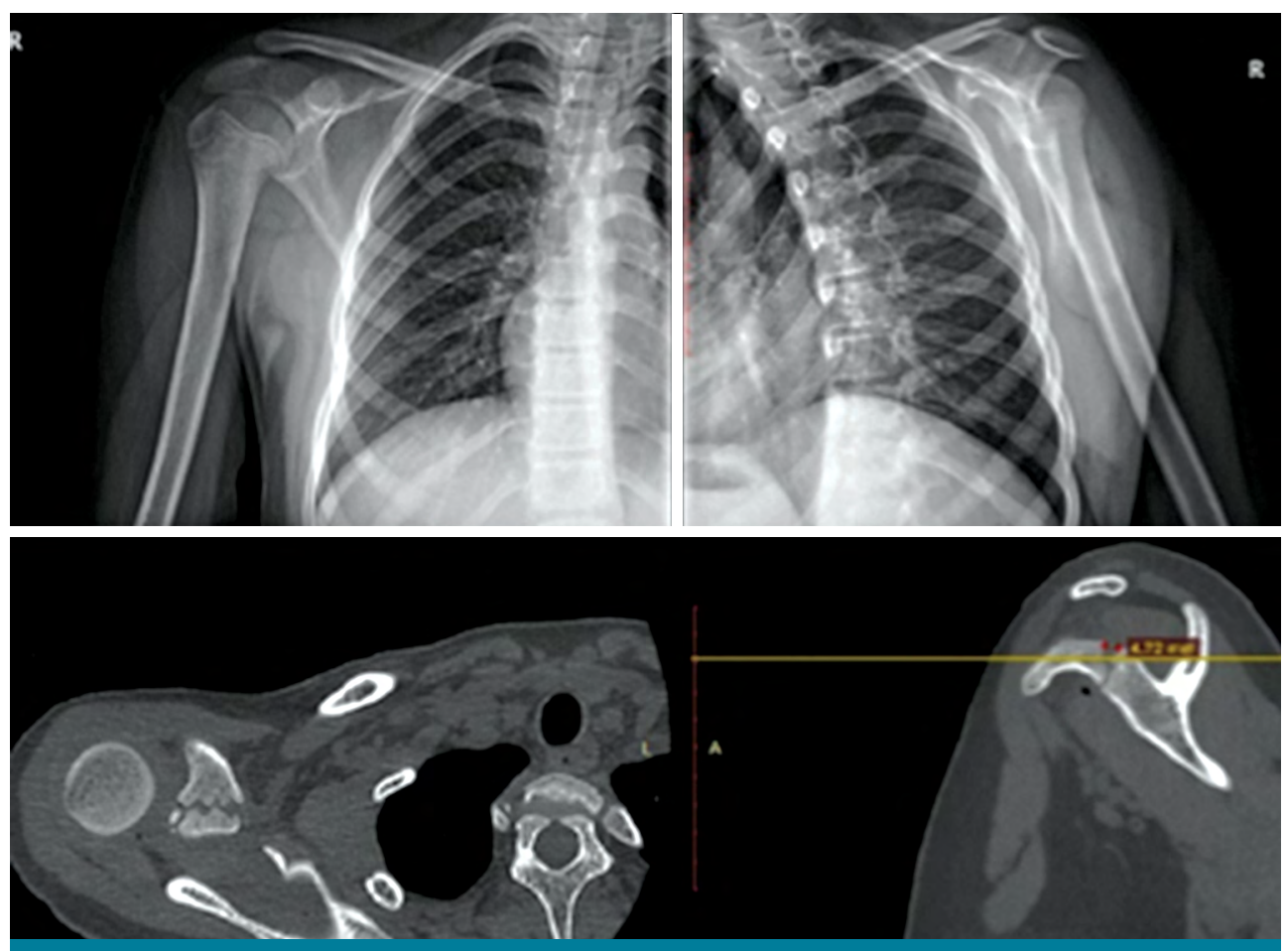

FIGURE 1. Top left and right frames contain anteroposterior and lateral radiographs of right shoulder obtained on admission, respectively. Bottom left and right frames contain computed tomography images in axial and sagittal planes which demonstrate isolated coracoid process fracture, respectively. Please note that distal fragment of coracoid process is displaced in anteroinferior direction and measured distance between bone fragments at the most superior part of fracture line is $4.7 \mathrm{~mm}$. 

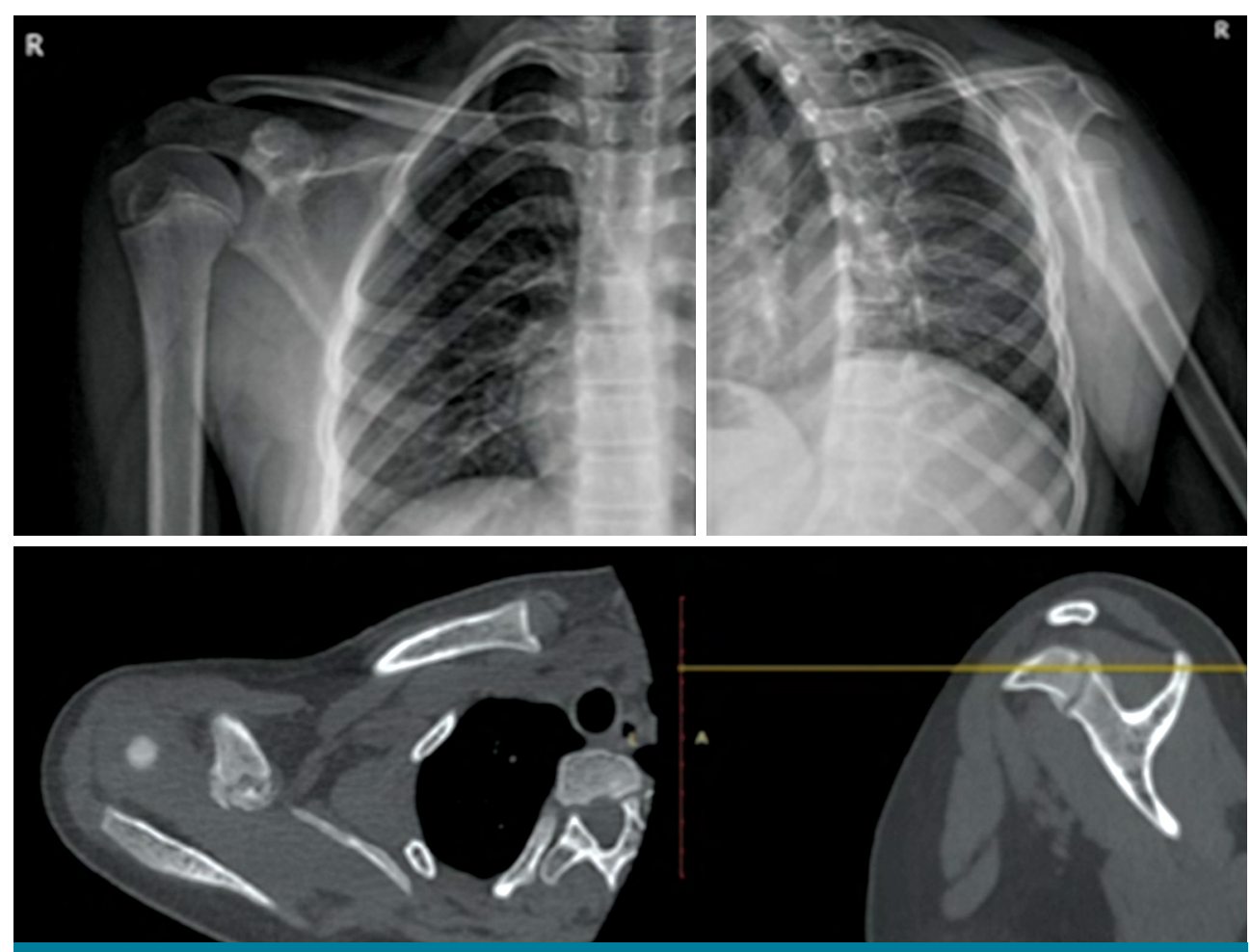

FIGURE 2. Top left and right frames contain anteroposterior and lateral radiographs of right shoulder, obtained four weeks after admission, respectively. Bottom left and right frames contain computed tomography images in axial and sagittal planes which demonstrate significant callus formation, respectively.

in conjunction with acromioclavicular joint dislocation.8 Glenohumeral joint dislocation, proximal humeral fracture, clavicular fracture or scapular body fracture may associate with coracoid fractures as well..$^{[1-3,8]}$ Isolated coracoid fractures are rare injuries. ${ }^{[1-6]}$

Controversy still exists on the management of ICFs. Indications for surgical management are symptomatic non-union, fracture displacement of more than $1 \mathrm{~cm}$, ipsilateral scapular fracture and disruption in superior shoulder suspensory complex. ${ }^{[4,5]}$ The literature comprises several reports recommending conservative management for ICFs. Vaienti and Pogliacomi ${ }^{[9]}$ reported complete healing with conservative treatment in eight out of nine cases diagnosed with ICF. The authors emphasized

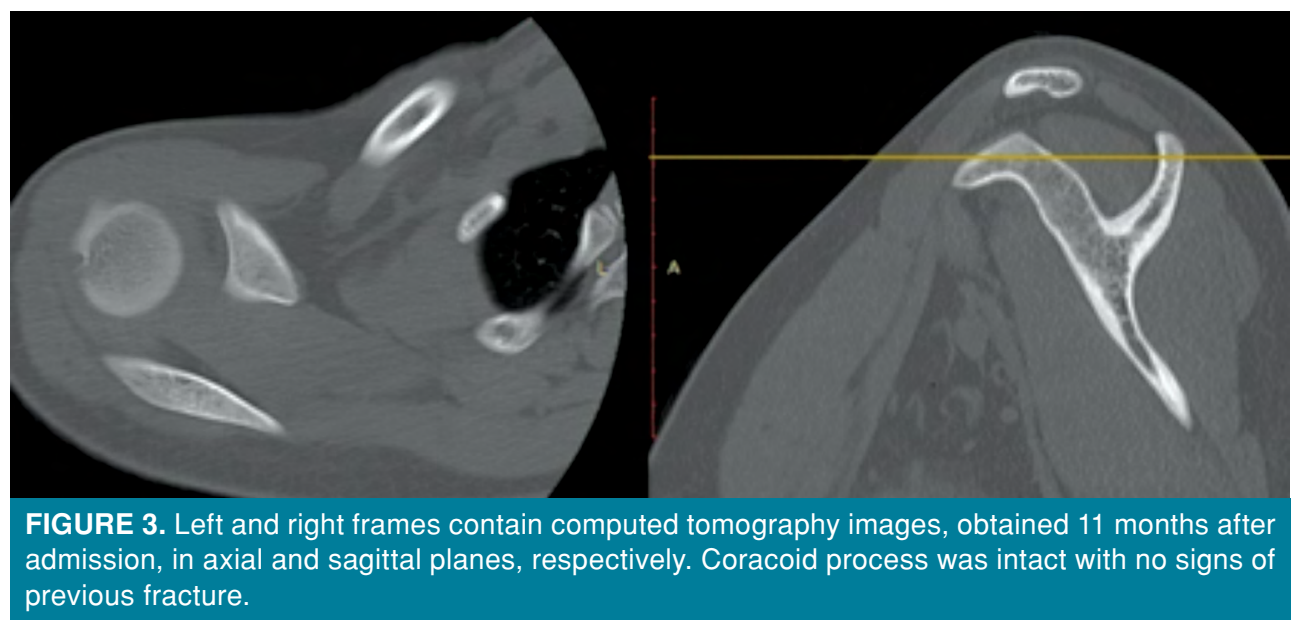


the need for suspicion and special projections to diagnose the fracture and recommended conservative treatment for the majority of ICFs. ${ }^{[9]}$ Guiral et al. ${ }^{[10]}$ mentioned satisfactory outcomes in a 49-year-old female patient treated conservatively for non-displaced isolated fracture of coracoid process. Ogawa et al. ${ }^{[1]}$ remarked no significant difference between the efficacy of surgical and non-surgical treatments with respect to follow-up of 45 cases sustaining coracoid process fractures and associated shoulder injuries. The authors recommended surgical treatment for patients with multiple injuries in shoulder girdle.

Recent reports on ICF indicate emerging tendency towards surgical management of these injuries, even in skeletally immature patients. ${ }^{[4-6]}$ Archik et al. ${ }^{[4]}$ presented an adolescent with minimally displaced coracoid fracture at distal part of the process. The authors reported favorable outcomes accomplished performing fracture fixation with cannulated screw. Currently, the preferred surgical procedure for surgical management of ICF in adults as well as skeletally immature patients is open reduction of fracture and fixation with cannulated screw. ${ }^{[4-6]}$ Besides, Kennedy et al. ${ }^{[11]}$ described a novel technique for the fixation of severely displaced ICFs. The authors reported successful outcomes for the repair of the chronic injury, performed with suture anchor fixation, in an adult.

Isolated coracoid fractures can easily be

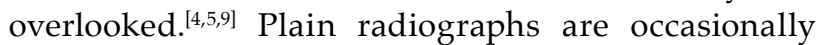
insufficient to demonstrate the fracture. Computed tomography is a valuable radiologic modality in the diagnosis of coracoid fractures as well as measurement of coracoid fracture displacement. ${ }^{[4-6]}$ The injury of the present case had been initially overlooked which is attributable to vague symptoms and signs as well as incompetency of plain radiographs to indicate the lesion clearly. Computed tomography images, obtained depending on the history of previous trauma and clinical suspicion, demonstrated the injury. The direction and amount of displacement were evaluated using CT images as well. Despite the disadvantage of radiation exposure, follow-up $\mathrm{CT}$, obtained at the end of fourth week, confirmed osseous union. Thus, an intensive rehabilitation protocol was commenced with confidence.

Isolated coracoid fractures are even rarer in skeletally immature population. ${ }^{[12]}$ Chitre et al..$^{[12]}$ presented two adolescent patients, each diagnosed with ICF, one with mild displacement and the other without any displacement. The authors reported favorable outcomes with conservative treatment of the injuries. Despite considerable displacement as depicted on the CT sections, conservative treatment of the present case achieved excellent clinical and radiologic outcome. Besides, external support was used within a relatively short period of time during which the symptoms of the patient were quite relieved.

Standard shoulder radiographs may fail to demonstrate ICF. Computed tomography imaging of injured shoulders is helpful to overcome missing the injury as well as to detect and quantify fracture displacement. Conservative treatment of Eyres type 2 ICF is capable of producing favorable radiologic and clinical outcomes in adolescents, even in the presence of considerable amount of fracture displacement.

\section{Declaration of conflicting interests}

The authors declared no conflicts of interest with respect to the authorship and/or publication of this article.

\section{Funding}

The authors received no financial support for the research and/or authorship of this article.

\section{REFERENCES}

1. Ogawa K, Yoshida A, Takahashi M, Ui M. Fractures of the coracoid process. J Bone Joint Surg [Br] 1997;79:17-9.

2. Eyres KS, Brooks A, Stanley D. Fractures of the coracoid process. J Bone Joint Surg [Br] 1995;77:425-8.

3. McGinnis M, Denton JR. Fractures of the scapula: a retrospective study of 40 fractured scapulae. J Trauma 1989;29:1488-93.

4. Archik S, Nanda SN, Tripathi S, Choudhari A, Rajadhyaksha H. An isolated displaced fracture of the coracoid process treated with open reduction and internal fixation - a case report and review of literature. J Orthop Case Rep 2016;6:37-9.

5. Güleç A, Kütahya H, Göncü RG, Toker S. Isolated fracture of the coracoid process. Case Rep Orthop 2014;2014:482130.

6. Li CH, Skalski MR, Matcuk GR Jr, Patel DB, Gross JS, Tomasian A, et al. Coracoid process fractures: anatomy, injury patterns, multimodality imaging, and approach to management. Emerg Radiol 2019;26:449-58.

7. Atik OŞ. Which articles do we prefer to publish? Eklem Hastalik Cerrahisi 2018;29:1.

8. Pedersen V, Prall WC, Ockert B, Haasters F. Non-operative treatment of a fracture to the coracoid process with acromioclavicular dislocation in an adolescent. Orthop Rev (Pavia) 2014;6:5499.

9. Vaienti E, Pogliacomi F. Delayed diagnosis of isolated coracoid process fractures: results of 9 cases treated conservatively. Acta Biomed 2012;83:138-46.

10. Guiral J, Real JL, Curto JM. Isolated fracture of the coracoid process of the scapula. Acta Orthop Belg 1996;62:60-1.

11. Kennedy NI, Ferrari MB, Godin JA, Sanchez G, Provencher MT. Repair of an isolated coracoid fracture with suture anchor fixation. Arthrosc Tech 2017;6:e1715-e9.

12. Chitre AR, Divecha HM, Hakimi M, Marynissen HA. Traumatic isolated coracoid fractures in the adolescent. Case Rep Orthop 2012;2012:371627. 